\title{
CRITICAL LENGTH FOR A QUENCHING PROBLEM WITH NONLOCAL SINGULARITY
}

\section{Go-Jien Chen and Jong-Shenq Guo}

\begin{abstract}
We study an initial-boundary-value problem for a nonlocal semilinear heat equation. By studying the existence and nonexistence of its stationary solutions, we obtain the critical length(s) of the original parabolic equation. Some peculiar features of critical lengths are different from the classical results, due to the nonlocal singularity.
\end{abstract}

\section{Introduction}

In this paper, we study the following initial-boundary-value problem for the nonlocal semilinear heat equation

$$
w_{s}=w_{y y}+\|w(\cdot, s)\|^{q}(1-w)^{-\beta}, \quad 0<y<a, \quad s>0,
$$

with zero Dirichlet boundary condition and with the initial condition

$$
w(y, 0)=w_{0}(y), \quad 0<y<a,
$$

where $a>0, q>0, \beta>0,0 \leq w_{0}(y)<1$ for $y \in[0, a]$, and the norm $\|\cdot\|$ is defined by

$$
\|w(\cdot, s)\|=\int_{0}^{a}|w(y, s)| d y .
$$

Note that by the standard parabolic theory, there exists a unique classical solution for a small time interval for the above initial-boundary-value problem. The solution $w$ of (1.1) is said to be quenching if the maximum of $w(\cdot, s)$ reaches 1 in some finite time. In this case, the equation becomes singular. It is well known that the stationary solutions of (1.1) play an important role in studying the quenching problem. According to the classical concept, a number $a^{*}$ is called a critical length for (1.1) if there are stationary solutions when $a<a^{*}$ and no stationary solutions when $a>a^{*}$ (cf. [1]). Our main purpose is to study the critical length(s) of this nonlocal problem.

First, we make the following transformation. Set

$$
u(x, t)=w(y, s), \quad y=a x, \quad s=a^{2} t .
$$

Then equation (1.1) becomes

$$
u_{t}=u_{x x}+\varepsilon\|u(\cdot, t)\|^{q}(1-u)^{-\beta}, \quad 0<x<1, \quad t>0,
$$

Received October 7, 1997, revised March 6, 1998.

1991 Mathematics Subject Classification: 35K20, 35K55, 35K60, 34B15 .

Key words and phrases: nonlocal semilinear heat equation, stationary solution, quenching, critical length . 
where

$$
\varepsilon=a^{2+q}, \quad\|u(\cdot, t)\|=\int_{0}^{1}|u(x, t)| d x .
$$

The quenching problem for the equation (1.2) was studied by Deng [2] for the case $\beta=1$ and $q>0$, and by Levine [3] for the case $\beta>0$ and $q=0$.

Therefore, the main subject of this paper is to study the existence and nonexistence of stationary solutions of (1.2). We are concerned with the nonnegative classical solution of the following problem:

$$
\begin{aligned}
& v^{\prime \prime}+\varepsilon\|v\|^{q}(1-v)^{-\beta}=0, \quad 0<x<1, \\
& v(0)=v(1)=0
\end{aligned}
$$

where $\|v\|=\int_{0}^{1} v(x) d x$. Note that $v \equiv 0$ is always a solution of $(\mathrm{S})$. By the maximum principle, any nontrivial nonnegative solution of $(\mathrm{S})$ must be positive in $(0,1)$. In the sequel, when we refer to a solution, we will mean a nontrivial positive solution.

When $\beta=1$, it was claimed in [2] that there is a unique positive solution of (S) for each given $\varepsilon>0$, if $q \geq 2$. But there is a gap in the proof of this result for $q>2$. Therefore, the case $q>2$ is still open. See the detailed explanations in Section 3 below.

In this paper, we shall restrict our attention to the case where $\beta \leq 1$. We summarize the main results of this paper as follows.

1. Suppose that $\beta=1$. Then the following hold:

(a) For $q=1$, there are no positive stationary solutions if $\varepsilon \geq 12$; and there is exactly one positive stationary solution if $\varepsilon<12$.

(b) If $1<q \leq 2+2 \theta_{0}^{2}$, then there is exactly one positive stationary solution for $\varepsilon>0$. (Here $\theta_{0}$ is a positive constant defined in Lemma 3.2 below.)

2. Suppose that $0<\beta<1$. Then the following hold:

(a) For $q=1$, there are no positive stationary solutions if $\varepsilon \geq 12$ or $0<\varepsilon \leq$ $4(1-\beta)(3+\beta) /(1+\beta)^{2}$, and there is exactly one positive stationary solution if $4(1-\beta)(3+\beta) /(1+\beta)^{2}<\varepsilon<12$.

(b) For $1<q \leq 2$, there is exactly one positive stationary solution if $\varepsilon>2^{3-q}(1-\beta)$ $(3+\beta) /(1+\beta)^{2}$, and there are no positive stationary solutions if $0<\varepsilon \leq 2^{3-q}(1-\beta)$ $(3+\beta) /(1+\beta)^{2}$.

The stability analysis for the above stationary solutions follows the same line as in [2] and [3], so that some quenching criteria and/or global existence results can be readily given (cf. [2] and [3]).

We make some remarks on the critical length(s) of the equation (1.1). From the above results, we see that there is a unique critical length for the case $\beta=1$ and $1 \leq q \leq 2+2 \theta_{0}^{2}$ and the case $0<\beta<1$ and $1<q \leq 2$. The critical length is either finite or infinite. Notice that in the case $0<\beta<1$ and $1<q \leq 2$, the critical length has the reverse meaning, namely, there are stationary solutions when $a>a^{*}$ and no stationary solutions when $a<a^{*}$. Furthermore, for the case $0<\beta<1$ and $q=1$, there are two critical lengths. There is a unique stationary solution when the length $a$ is between these two critical values. This peculiar feature is different from the classical results due to the nonlocal singularity. 
We organize this paper as follows. In Section 2, we give some preliminaries which will be used later. In Section 3 , the case $\beta=1$ is studied. In Section 4 , the case $0<\beta<1$ is treated for the range $1 \leq q \leq 2$.

\section{Preliminaries}

Let $v(x)$ be a nontrivial nonnegative classical solution of

$$
v^{\prime \prime}+\varepsilon\|v\|^{q}(1-v)^{-\beta}=0, \quad 0<x<1,
$$

with $v(0)=v(1)=0$, for certain choices of $\varepsilon, q, \beta$, with $\varepsilon>0, q>0$, and $\beta>0$. Then by the strong maximum principle, we have $v(x)>0$ and so $v^{\prime \prime}<0$ on $(0,1)$. Thus $v$ has exactly one maximum in $(0,1)$, say at $\xi$. Let

$$
F_{1}(s)= \begin{cases}-\ln (1-s) & \text { if } \beta=1 \\ (\beta-1)^{-1}(1-s)^{1-\beta} & \text { if } \beta \neq 1\end{cases}
$$

and

$$
\tilde{G}_{1}(\mu)=\int_{0}^{\mu} \frac{1}{\sqrt{F_{1}(\mu)-F_{1}(\eta)}} d \eta
$$

where $\mu=v(\xi)<1$. Then it follows from [2] that $\xi=1 / 2$, and $\tilde{G}_{1}(\mu)$ can be represented as

$$
\tilde{G}_{1}(\mu)=\sqrt{\varepsilon / 2}\|v\|^{q / 2} .
$$

In order to derive a relation between $\varepsilon$ and $\mu$ which is independent of $\|v\|$, we borrow an idea from Deng [2], and let

$$
y=\int_{0}^{x}(1-v(s)) d s, \quad g(y)=(1-v(x(y)))^{2} .
$$

Then $g$ satisfies

$$
\begin{aligned}
& g_{y y}-2 \varepsilon\|v\|^{q} g^{-(\beta+1) / 2}=0, \quad 0<y<Y, \\
& g(0)=g(Y)=1, \quad Y=1-\|v\| .
\end{aligned}
$$

Setting $z=y / Y$ and $h(z)=1-g(y)$, we obtain

$$
\begin{aligned}
& h_{z z}+2 \varepsilon\|v\|^{q}(1-\|v\|)^{2}(1-h)^{-(\beta+1) / 2}=0, \quad 0<z<1, \\
& h(0)=h(1)=0 .
\end{aligned}
$$

Define

$$
F_{2}(s)= \begin{cases}-\ln (1-s) & \text { if } \beta=1 \\ 2(\beta-1)^{-1}(1-s)^{(1-\beta) / 2} & \text { if } \beta \neq 1\end{cases}
$$

and

$$
\tilde{G}_{2}(\lambda)=\int_{0}^{\lambda} \frac{1}{\sqrt{F_{2}(\lambda)-F_{2}(\eta)}} d \eta
$$

where $\lambda=\max _{0 \leq z \leq 1} h(z)$. Note that $\lambda=2 \mu-\mu^{2}$ with $\mu=\max _{0 \leq x \leq 1} v(x)$. By similar reasoning, we obtain

$$
\tilde{G}_{2}(\lambda)=\sqrt{\varepsilon}\|v\|^{q / 2}(1-\|v\|) .
$$


Then it follows from (2.3) and (2.5) that

$$
\|v\|=\frac{\sqrt{2} \tilde{G}_{1}(\mu)-\tilde{G}_{2}(\lambda)}{\sqrt{2} \tilde{G}_{1}(\mu)}
$$

Substituting (2.6) into (2.3), we obtain

where

$$
\varepsilon=\tilde{K}_{q}(\mu)
$$

$$
\tilde{K}_{q}(\mu)=2\left(\frac{\sqrt{2}\left(\tilde{G}_{1}(\mu)\right)^{1+2 / q}}{\sqrt{2} \tilde{G}_{1}(\mu)-\tilde{G}_{2}(\lambda)}\right)^{q} .
$$

Conversely, for a fixed $\beta>0$, a given $\mu \in(0,1)$, and a $q>0$, let $\varepsilon=\tilde{K}_{q}(\mu)$. We define $v(x)$ implicitly by

$$
\int_{0}^{v(x)} \frac{1}{\sqrt{F_{1}(\mu)-F_{1}(\eta)}} d \eta=2 \tilde{G}_{1}(\mu) x
$$

for $0 \leq x \leq 1 / 2$, and $v(x)=v(1-x)$ if $1 / 2<x \leq 1$. Then, by reasoning similar to the above, it is easy to show that $v(x)$ is a solution of $(\mathrm{S})$.

Therefore, studying the existence of stationary solutions is equivalent to studying the behavior of the function $\tilde{K}_{q}$ in $(0,1)$. First, the following lemma shows that the function $\tilde{K}_{q}$ is well-defined in $(0,1)$. Since the proof can be found in [2], we omit it.

Lemma 2.1. $\sqrt{2} \tilde{G}_{1}(\mu)-\tilde{G}_{2}(\lambda)>0$ for $\mu \in(0,1)$.

Since $\tilde{G}_{1}(\mu)>0$ for $\mu \in(0,1)$, this implies that $\tilde{K}_{q}(\mu)>0$ for $\mu \in(0,1)$. To investigate the behavior of $\tilde{K}_{q}(\mu)$ on $(0,1)$, we use the transformation

$$
\theta=\theta(\mu)= \begin{cases}{[-\ln (1-\mu)]^{1 / 2}} & \text { if } \beta=1, \\ {\left[(1-\mu)^{\beta-1}-1\right]^{1 / 2}} & \text { if } 0<\beta<1,\end{cases}
$$

and let $G_{1}(\theta)=\tilde{G}_{1}(\mu), G_{2}(\theta)=\tilde{G}_{2}(\lambda)$, and $K_{q}(\theta)=\tilde{K}_{q}(\mu)$. Note that $\mu \in(0,1)$ if and only if $\theta \in(0, \infty)$ and $\theta(\mu)$ is increasing in $\mu$ for any $\beta \in(0,1]$.

For convenience, we also define

$$
\begin{aligned}
A(\theta) & =\int_{0}^{\theta} e^{\sigma^{2}} d \sigma, \\
B(\theta) & =\int_{0}^{\theta} e^{2 \sigma^{2}} d \sigma, \\
A_{+}(\theta) & =\int_{0}^{\theta}\left(1+\sigma^{2}\right)^{\frac{\beta}{1-\beta}} d \sigma, \\
B_{+}(\theta) & =\int_{0}^{\theta}\left(1+\sigma^{2}\right)^{\frac{1+\beta}{1-\beta}} d \sigma .
\end{aligned}
$$

When $\beta=1$, we have

$$
\begin{aligned}
& G_{1}(\theta)=2 e^{-\theta^{2}} A(\theta) \\
& G_{2}(\theta)=2 \sqrt{2} e^{-2 \theta^{2}} B(\theta) \\
& K_{q}(\theta)=8 e^{-2 \theta^{2}} A^{q+2}(\theta)\left[A(\theta)-e^{-\theta^{2}} B(\theta)\right]^{-q} .
\end{aligned}
$$


For $0<\beta<1$, we have

$$
\begin{aligned}
& G_{1}(\theta)=\frac{2}{\sqrt{1-\beta}}\left(1+\theta^{2}\right)^{\frac{\beta+1}{2(\beta-1)}} A_{+}(\theta), \\
& G_{2}(\theta)=\frac{2 \sqrt{2}}{\sqrt{1-\beta}}\left(1+\theta^{2}\right)^{\frac{\beta+3}{2(\beta-1)}} B_{+}(\theta) . \\
& K_{q}(\theta)=\frac{8}{1-\beta} \frac{\left(1+\theta^{2}\right)^{\frac{\beta+1}{\beta-1}}\left[A_{+}(\theta)\right]^{q+2}}{\left[A_{+}(\theta)-\left(1+\theta^{2}\right)^{\frac{1}{\beta-1}} B_{+}(\theta)\right]^{q}} .
\end{aligned}
$$

We will investigate the function $K_{q}(\theta)$ in the following sections.

\section{The case $\beta=1$}

First, we recall from [2] that

$$
\lim _{\theta \rightarrow \infty} K_{q}(\theta)=0 \quad \text { for } q>0
$$

and

$$
\lim _{\theta \rightarrow 0^{+}} K_{q}(\theta)= \begin{cases}\infty & \text { for } q>1 \\ 12 & \text { for } q=1 \\ 0 & \text { for } q<1\end{cases}
$$

Using (2.9) and (2.10), the following lemma follows from Lemma 2.1 directly.

Lemma 3.1. $A(\theta)-e^{-\theta^{2}} B(\theta)>0$ on $(0, \infty)$.

The following lemma can be found in [3].

Lemma 3.2. There is $a \theta_{0} \in(0, \infty)$ such that

$$
\begin{array}{ll}
1>2 \theta e^{-\theta^{2}} A(\theta) & \text { for } \theta \in\left(0, \theta_{0}\right), \\
1<2 \theta e^{-\theta^{2}} A(\theta) & \text { for } \theta \in\left(\theta_{0}, \infty\right) .
\end{array}
$$

By (2.11), the derivative $K_{q}^{\prime}(\theta)$ can be calculated as

$$
K_{q}^{\prime}(\theta)=8\left[A(\theta)-e^{-\theta^{2}} B(\theta)\right]^{-(q+1)} A^{q+1}(\theta) e^{-\theta^{2}} J_{q}(\theta)
$$

where

$$
J_{q}(\theta)=(2+q)\left(A(\theta)-e^{-\theta^{2}} B(\theta)\right)-4 \theta e^{-\theta^{2}} A^{2}(\theta)+2(2-q) \theta e^{-2 \theta^{2}} A(\theta) B(\theta) .
$$

Note that by (3.3), the sign of $K_{q}^{\prime}(\theta)$ is the same as $J_{q}(\theta)$. Now, we will determine the sign of $J_{q}(\theta)$ in the following. Differentiating $J_{q}(\theta)$ in (3.4), we get

$$
J_{q}^{\prime}(\theta)=-2 \theta J_{q}(\theta)-4 \theta e^{-\theta^{2}} A(\theta)\left(A(\theta)-e^{-\theta^{2}} B(\theta)\right)+2 e^{-\theta^{2}} B(\theta) h_{1}(\theta)
$$

where

$$
h_{1}(\theta)=(2-q) \theta\left[1-2 \theta e^{-\theta^{2}} A(\theta)\right]-q e^{-\theta^{2}} A(\theta) .
$$

Lemma 3.3. For $1 \leq q \leq 2$, we have

$$
h_{1}(\theta)<0 \quad \text { for } \theta \in(0, \infty) .
$$


Proof. Since

$$
h_{1}^{\prime}(\theta)=-2 \theta h_{1}(\theta)-4 \theta e^{-\theta^{2}} A(\theta)(2-q)+2(1-q),
$$

we obtain

$$
\left[e^{\theta^{2}} h_{1}(\theta)\right]^{\prime}=\left[-4 \theta e^{-\theta^{2}} A(\theta)(2-q)+2(1-q)\right] e^{\theta^{2}} .
$$

For $1 \leq q \leq 2$, we know that $e^{\theta^{2}} h_{1}(\theta)$ is a strictly decreasing function. Since

$$
\lim _{\theta \rightarrow 0^{+}} e^{\theta^{2}} h_{1}(\theta)=0 \text {, }
$$

the lemma follows.

We are ready to prove a part of the conjecture in [2] as follows.

Theorem 3.4. Suppose that $\beta=1$. Then the following hold:

1. For $q=1$, there are no positive stationary solutions if $\varepsilon \geq 12$, and there is exactly one positive stationary solution if $\varepsilon<12$.

2. If $1<q \leq 2$, then there is exactly one positive stationary solution for $\varepsilon>0$.

Proof. By (3.5) and Lemma 3.3, we have

$$
\left[e^{\theta^{2}} J_{q}(\theta)\right]^{\prime}=\left[-4 \theta e^{-\theta^{2}} A(\theta)\left(A(\theta)-e^{-\theta^{2}} B(\theta)\right)+2 e^{-\theta^{2}} B(\theta) h_{1}(\theta)\right] e^{\theta^{2}}<0 .
$$

This implies that $e^{\theta^{2}} J_{q}(\theta)$ is a strictly decreasing function in $(0, \infty)$ for $1 \leq q \leq 2$. Since $\lim _{\theta \rightarrow 0^{+}} e^{\theta^{2}} J_{q}(\theta)=0$, we have

$$
J_{q}(\theta)<0, \quad \text { for } \theta \in(0, \infty) \text { and } 1 \leq q \leq 2 .
$$

Hence the theorem follows from (3.1), (3.2), and (3.7).

In [2], the author claimed that there is exactly one positive solution for $\varepsilon>0$, $q>2$. Indeed, the proof of this case is not complete since the $\operatorname{limit}_{\mu \rightarrow 1^{-}} J_{2}^{\prime}(\mu)$ is $-\infty$ instead of $+\infty$ as stated in the proof of Lemma 3.3 in [2]. From [2], we have

$$
\begin{aligned}
& J_{2}(\mu)=\left(\sqrt{F(\mu)}-\frac{1}{2 \sqrt{F(\mu)}}\right) G(\mu)-1 \\
& J_{2}^{\prime}(\mu)=f(\mu)\left[-J_{2}(\mu)+\frac{1}{2 \sqrt{F(\mu)}}\left(G(\mu)-\frac{1}{\sqrt{F(\mu)}}\right)+\frac{G(\mu)}{4(F(\mu))^{3 / 2}}\right] .
\end{aligned}
$$

Substituting $f(\mu)=e^{\theta^{2}}, F(\mu)=\theta^{2}, G(\mu)=2 e^{-\theta^{2}} A(\theta)$ into (3.9), we obtain

$$
J_{2}^{\prime}(\mu)=\left(1-\frac{1}{2} \theta^{-2}\right) e^{\theta^{2}}\left(1-2 \theta e^{-\theta^{2}} A(\theta)\right)+\left(1+\frac{1}{2} \theta^{-2}\right) \theta^{-1} A(\theta) .
$$

By (3.10), we compute

$$
\lim _{\mu \rightarrow 1^{-}} J_{2}^{\prime}(\mu)=\lim _{\theta \rightarrow \infty}\left(1-\frac{1}{2} \theta^{-2}\right) e^{\theta^{2}}\left(1-2 \theta e^{-\theta^{2}} A(\theta)\right)+\left(1+\frac{1}{2} \theta^{-2}\right) \theta^{-1} A(\theta) .
$$

Using integration by parts, we obtain

$$
\begin{gathered}
A(\theta)=e^{\theta^{2}}\left(\frac{1}{2} \theta^{-1}+\frac{1}{4} \theta^{-3}+\frac{3}{8} \theta^{-5}+\frac{15}{16} \theta^{-7}+\frac{105}{32} \theta^{-9}+\cdots\right) \\
+\int_{0}^{1} e^{\sigma^{2}} d \sigma-e\left(\frac{1}{2}+\frac{1}{4}+\frac{3}{8}+\frac{15}{16}+\cdots\right) .
\end{gathered}
$$


Substituting (3.12) into (3.11), we conclude that

$$
\lim _{\mu \rightarrow 1^{-}} J_{2}^{\prime}(\mu)=\lim _{\theta \rightarrow \infty} e^{\theta^{2}}\left(-\theta^{-6}-\frac{9}{2} \theta^{-8}-\cdots\right)=-\infty .
$$

Because $\lim _{\mu \rightarrow 1^{-}} J_{2}^{\prime}(\mu)=-\infty$, the proof of Lemma 3.3 in [2] is not correct. Accordingly the proof of Theorem 3.4 in [2] is also not complete. Therefore, the case $q>2$ is still open. Here we will prove that there is exactly one positive stationary solution for the case $2<q \leq 2+2 \theta_{0}^{2}$ where $\theta_{0}$ is the constant given in Lemma 3.2.

Theorem 3.5. Suppose that $\beta=1$. If $2<q \leq 2+2 \theta_{0}^{2}$, then for every $\varepsilon>0$, there is exactly one positive stationary solution.

Proof. By (3.6) and Lemma 3.2, we have

$$
h_{1}(\theta)<0 \text { if } q>2 \text { and } \theta \in\left(0, \theta_{0}\right] .
$$

Hence, by (3.5), we obtain

$$
J_{q}(\theta)<0 \text { if } q>2 \text { and } \theta \in\left(0, \theta_{0}\right] .
$$

We want to claim that

$$
J_{q}(\theta)<0 \text { if } 2<q \leq 2+2 \theta_{0}^{2} \text { and } \theta \in\left(\theta_{0}, \infty\right) .
$$

From (3.6), the definition of $h_{1}(\theta)$, we have

$$
h_{1}^{\prime}(\theta)=-2 \theta h_{1}(\theta)+h_{2}(\theta)
$$

where

$$
h_{2}(\theta)=2(2-q)\left(1-2 \theta e^{-\theta^{2}} A(\theta)\right)-2 .
$$

From (3.16), we compute

$$
h_{2}^{\prime}(\theta)=-2 \theta h_{2}(\theta)-4 f(\theta)
$$

where

$$
f(\theta)=\theta-(q-2) e^{-\theta^{2}} A(\theta) .
$$

By (3.18), we have

$$
f^{\prime}(\theta)=1-(q-2)\left(1-2 \theta e^{-\theta^{2}} A(\theta)\right)
$$

so

$$
f^{\prime}(\theta)>0 \quad \text { for } \theta \in\left(\theta_{0}, \infty\right) \text { and } q>2
$$

Since

$$
1=2 \theta_{0} e^{-\theta_{0}^{2}} A\left(\theta_{0}\right),
$$

we have

$$
f\left(\theta_{0}\right)=\theta_{0}-(q-2) \frac{1}{2 \theta_{0}} .
$$

If $2<q \leq 2+2 \theta_{0}^{2}$, then $f\left(\theta_{0}\right) \geq 0$ and so

$$
f(\theta)>0 \quad \text { for } \theta \in\left(\theta_{0}, \infty\right) \text {. }
$$

Now, suppose that $2<q \leq 2+2 \theta_{0}^{2}$. Substituting (3.19) into (3.17), we get

$$
\left(e^{\theta^{2}} h_{2}(\theta)\right)^{\prime}<0 \quad \text { for } \theta \in\left(\theta_{0}, \infty\right)
$$


Since $e^{\theta_{0}^{2}} h_{2}\left(\theta_{0}\right)=-2 e^{\theta_{0}^{2}}<0$, it follows that

$$
h_{2}(\theta)<0 \quad \text { for } \theta \in\left(\theta_{0}, \infty\right) .
$$

Substituting (3.20) into (3.15), we obtain

$$
\left(e^{\theta^{2}} h_{1}(\theta)\right)^{\prime}<0 \quad \text { for } \theta \in\left(\theta_{0}, \infty\right) .
$$

Since $e^{\theta_{0}^{2}} h_{1}\left(\theta_{0}\right)=-q A\left(\theta_{0}\right)<0$, this implies that

$$
h_{1}(\theta)<0 \quad \text { for } \theta \in\left(\theta_{0}, \infty\right) .
$$

Substituting (3.21) into (3.5), we have

$$
\left(e^{\theta^{2}} J_{q}(\theta)\right)^{\prime}<0 \quad \text { for } \theta \in\left(\theta_{0}, \infty\right) .
$$

Then, using the fact that $e^{\theta_{0}^{2}} J_{q}\left(\theta_{0}\right)<0,(3.14)$ follows. Therefore, the theorem follows from (3.13) and (3.14) directly.

\section{The case $0<\beta<1$}

First, from (2.14) and l'Hôpital's rule, we obtain

$$
\lim _{\theta \rightarrow \infty} K_{q}(\theta)=\frac{2^{3-q}(1-\beta)(3+\beta)^{q}}{(1+\beta)^{2}} \quad \text { for } q>0
$$

and

$$
\lim _{\theta \rightarrow 0^{+}} K_{q}(\theta)= \begin{cases}\infty & \text { for } q>1, \\ 12 & \text { for } q=1, \\ 0 & \text { for } 0<q<1 .\end{cases}
$$

Substituting (2.12) and (2.13) into Lemma 2.1, we obtain

Lemma 4.1. $A_{+}(\theta)-\left(1+\theta^{2}\right)^{\frac{1}{\beta-1}} B_{+}(\theta)>0$ for $\theta \in(0, \infty)$.

Also, from [3], we have

Lemma 4.2. There is a $\theta_{1} \in(0, \infty)$ such that

$$
\begin{array}{ll}
1>\frac{\beta+1}{1-\beta} \theta\left(1+\theta^{2}\right)^{\frac{1}{\beta-1}} A_{+}(\theta) & \text { for } \theta \in\left(0, \theta_{1}\right), \\
1<\frac{\beta+1}{1-\beta} \theta\left(1+\theta^{2}\right)^{\frac{1}{\beta-1}} A_{+}(\theta) & \text { for } \theta \in\left(\theta_{1}, \infty\right) .
\end{array}
$$

By (2.14), the derivative $K_{q}^{\prime}(\theta)$ can be calculated as

$$
K_{q}^{\prime}(\theta)=\frac{4\left(1-\theta^{2}\right)}{\theta}\left[\frac{A_{+}(\theta)}{A_{+}(\theta)-\left(1+\theta^{2}\right)^{\frac{1}{\beta-1}} B_{+}(\theta)}\right]^{q+1} J_{q}(\theta)
$$

where

$$
\begin{aligned}
J_{q}(\theta)=(2 & +q)\left(A_{+}(\theta)-\left(1+\theta^{2}\right)^{\frac{1}{\beta-1}} B_{+}(\theta)\right)-\frac{2(\beta+1)}{1-\beta}\left(1+\theta^{2}\right)^{\frac{1}{\beta-1}} \theta A_{+}^{2}(\theta) \\
& +\frac{2(\beta+1-q)}{1-\beta}\left(1+\theta^{2}\right)^{\frac{2}{\beta-1}} \theta A_{+}(\theta) B_{+}(\theta) .
\end{aligned}
$$


From (4.3), we compute

$$
\begin{aligned}
J_{q}^{\prime}(\theta)=- & \frac{2}{1-\beta} \theta\left(1+\theta^{2}\right)^{-1} J_{q}(\theta)-2\left(A_{+}(\theta)-\left(1+\theta^{2}\right)^{\frac{1}{\beta-1}} B_{+}(\theta)\right)\left(1+\theta^{2}\right)^{\frac{1}{\beta-1}} h(\theta) \\
& +\frac{2}{1-\beta}\left(1+\theta^{2}\right)^{\frac{2-\beta}{(\beta-1)}} B_{+}(\theta) h_{1}^{+}(\theta)
\end{aligned}
$$

where

$$
\begin{aligned}
h(\theta) & =\frac{1+\beta}{1-\beta} A_{+}(\theta)-\theta\left(1+\theta^{2}\right)^{\frac{\beta}{1-\beta}} \\
h_{1}^{+}(\theta) & =(2-q) \theta\left(1-\frac{\beta+1}{1-\beta} \theta\left(1+\theta^{2}\right)^{\frac{1}{\beta-1}} A_{+}(\theta)\right)-q\left(1+\theta^{2}\right)^{\frac{1}{\beta-1}} A_{+}(\theta) .
\end{aligned}
$$

Lemma 4.3. The function $h(\theta)>0$ for $\theta \in(0, \infty)$.

Proof. Since $h^{\prime}(\theta)=\frac{2 \beta}{1-\beta}\left(1+\theta^{2}\right)^{\frac{2 \beta-1}{1-\beta}}>0$ and $\lim _{\theta \rightarrow 0^{+}} h(\theta)=0$, the lemma follows.

We are ready to state and prove the following main theorem of this section.

Theorem 4.4. Suppose that $0<\beta<1$. Then the following hold:

1. For $q=1$, there are no positive stationary solutions if $\varepsilon \geq 12$ or $0<\varepsilon \leq 4(1-\beta)(3+\beta) /(1+\beta)^{2}$, and there is exactly one stationary solution if $4(1-\bar{\beta})(3+\beta) /(1+\beta)^{2}<\varepsilon<12$.

2. For $1<q \leq 2$, there is exactly one positive stationary solution if $\varepsilon>2^{3-q}(1-\beta)(3+\beta) /(1+\beta)^{2}$, and there are no positive stationary solutions if $0<\varepsilon \leq 2^{3-q}(1-\beta)(3+\beta) /(1+\beta)^{2}$.

Proof. From (4.6), we compute

$$
\begin{aligned}
\left(h_{1}^{+}(\theta)\right)^{\prime}=- & \frac{2}{1-\beta} \theta\left(1+\theta^{2}\right)^{-1} h_{1}^{+}(\theta)-\frac{2(\beta+1)}{1-\beta} \theta\left(1+\theta^{2}\right)^{\frac{1}{\beta-1}} A_{+}(\theta)(2-q) \\
& +2(1-q)+2 \theta^{2}\left(1+\theta^{2}\right)^{-1} .
\end{aligned}
$$

If $q=2$, then we have

$$
\left(h_{1}^{+}(\theta)\right)^{\prime}=-\frac{2}{1-\beta} \theta\left(1+\theta^{2}\right)^{-1} h_{1}^{+}(\theta)-\frac{2}{1+\theta^{2}} .
$$

This implies that

$$
\left[\left(1+\theta^{2}\right)^{\frac{1}{1-\beta}} h_{1}^{+}(\theta)\right]^{\prime}=\left[-\frac{2}{1+\theta^{2}}\right]\left(1+\theta^{2}\right)^{\frac{1}{1-\beta}}<0 \quad \text { for } \theta \in(0, \infty) .
$$

Since

$$
\lim _{\theta \rightarrow 0^{+}}\left(1+\theta^{2}\right)^{\frac{1}{1-\beta}} h_{1}^{+}(\theta)=0,
$$

we have $\left(1+\theta^{2}\right)^{\frac{1}{1-\beta}} h_{1}^{+}(\theta)<0$ for $\theta \in(0, \infty)$, and so $h_{1}^{+}(\theta)<0$ for $\theta \in(0, \infty)$.

If $q=1$, then we have

$$
\left(h_{1}^{+}(\theta)\right)^{\prime}=-\frac{2}{1-\beta} \theta\left(1+\theta^{2}\right)^{-1} h_{1}^{+}(\theta)-2 \theta\left(1+\theta^{2}\right)^{\frac{1}{\beta-1}} h(\theta) .
$$

By Lemma 4.3, we also conclude that $h_{1}^{+}(\theta)<0$ for $\theta \in(0, \infty)$. 
Therefore, by (4.4), we obtain

$$
J_{1}(\theta)<0 \quad \text { and } \quad J_{2}(\theta)<0 \quad \text { for } \theta \in(0, \infty) .
$$

By (4.6) and Lemma 4.2, we have

$$
h_{1}^{+}(\theta)<0 \quad \text { if } q \leq 2 \text { and } \theta \in\left[\theta_{1}, \infty\right) .
$$

It follows from (4.8), Lemma 4.3, and (4.4) that

$$
J_{q}(\theta)<0 \quad \text { if } q \leq 2 \text { and } \theta \in\left[\theta_{1}, \infty\right) .
$$

We rewrite $J_{q}(\theta)$ in (4.3) as

$$
J_{q}(\theta)=q \tilde{J}_{q}(\theta)
$$

where

$$
\begin{gathered}
\tilde{J}_{q}(\theta)=\left(1+\frac{2}{q}\right)\left(A_{+}(\theta)-\left(1+\theta^{2}\right)^{\frac{1}{\beta-1}} B_{+}(\theta)\right)\left(1-\frac{\beta+1}{1-\beta} \theta\left(1+\theta^{2}\right)^{\frac{1}{\beta-1}} A_{+}(\theta)\right) \\
-\theta\left(1+\theta^{2}\right)^{\frac{1}{\beta-1}} A_{+}(\theta)\left[\frac{3+\beta}{1-\beta}\left(1+\theta^{2}\right)^{\frac{1}{\beta-1}} B_{+}(\theta)-\frac{1+\beta}{1-\beta} A_{+}(\theta)\right] .
\end{gathered}
$$

From (4.10), we know that the sign of $J_{q}(\theta)$ is the same as $\tilde{J}_{q}(\theta)$. Therefore, by (4.9), we have

$$
\tilde{J}_{q}(\theta)<0 \quad \text { if } q \leq 2 \text { and } \theta \in\left[\theta_{1}, \infty\right) .
$$

If $\theta \in\left(0, \theta_{1}\right)$, then by (4.11) we know that $\tilde{J}_{q}(\theta)$ is a strictly decreasing function in $q$. Thus we have

$$
\tilde{J}_{q}(\theta)<\tilde{J}_{1}(\theta)<0 \quad \text { if } q>1 \text { and } \theta \in\left(0, \theta_{1}\right) .
$$

Therefore, by (4.7), (4.10), (4.12) and (4.13), we have

$$
J_{q}(\theta)<0 \quad \text { for } \theta \in(0, \infty) \text { and } 1 \leq q \leq 2 .
$$

Recall (4.1) and (4.2). Hence the theorem is proved.

Acknowledgments. This work is partially supported by the National Science Council of the Republic of China under contract NSC 86-2115-M-003-001.

\section{References}

1. C. Y. Chan, Quenching phenomena for reaction-diffusion equations, Proc. Dynamic Systems and Applications 1 (1994), 51-58.

2. K. Deng, Dynamical behavior of solutions of a semilinear heat equation with nonlocal singularity, SIAM J. Math. Anal. 26 (1995), 98-111.

3. H. A. Levine, Quenching, nonquenching, and beyond quenching for solution of some parabolic equations, Ann. Mat. Pura Appl. 155 (1989), 243-260.

Department of Mathematics, National TAiwan Normal University, TAipei 117, TAiwan

E-mail: jsguo@math.ntnu.edu.tw 